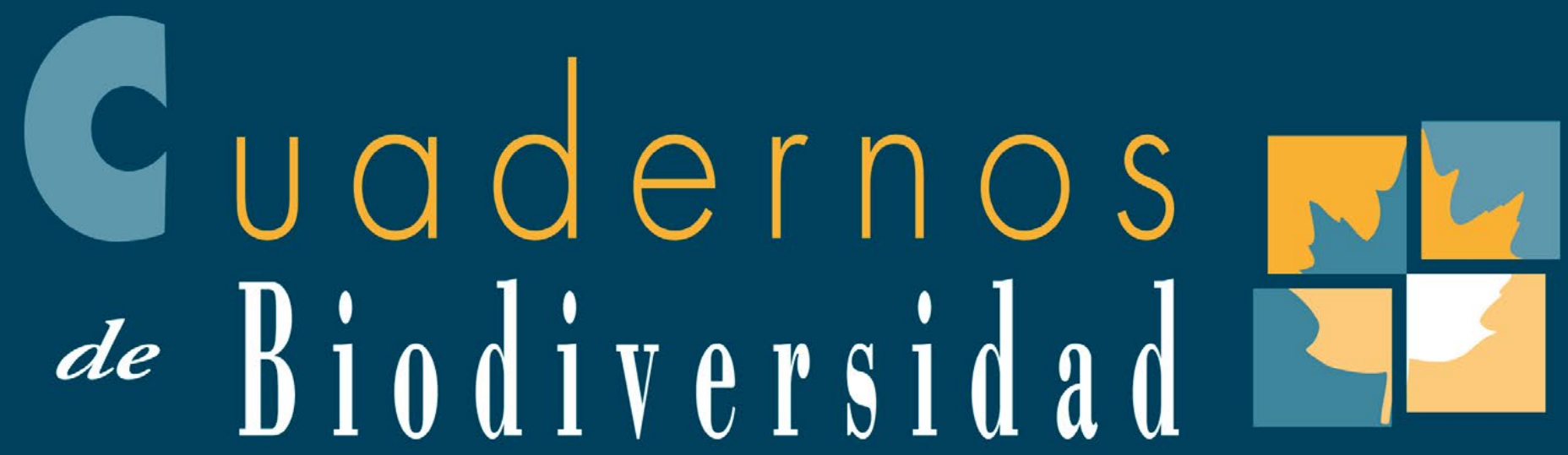

Cuadernos de biodiversidad número $49 \cdot 2015$
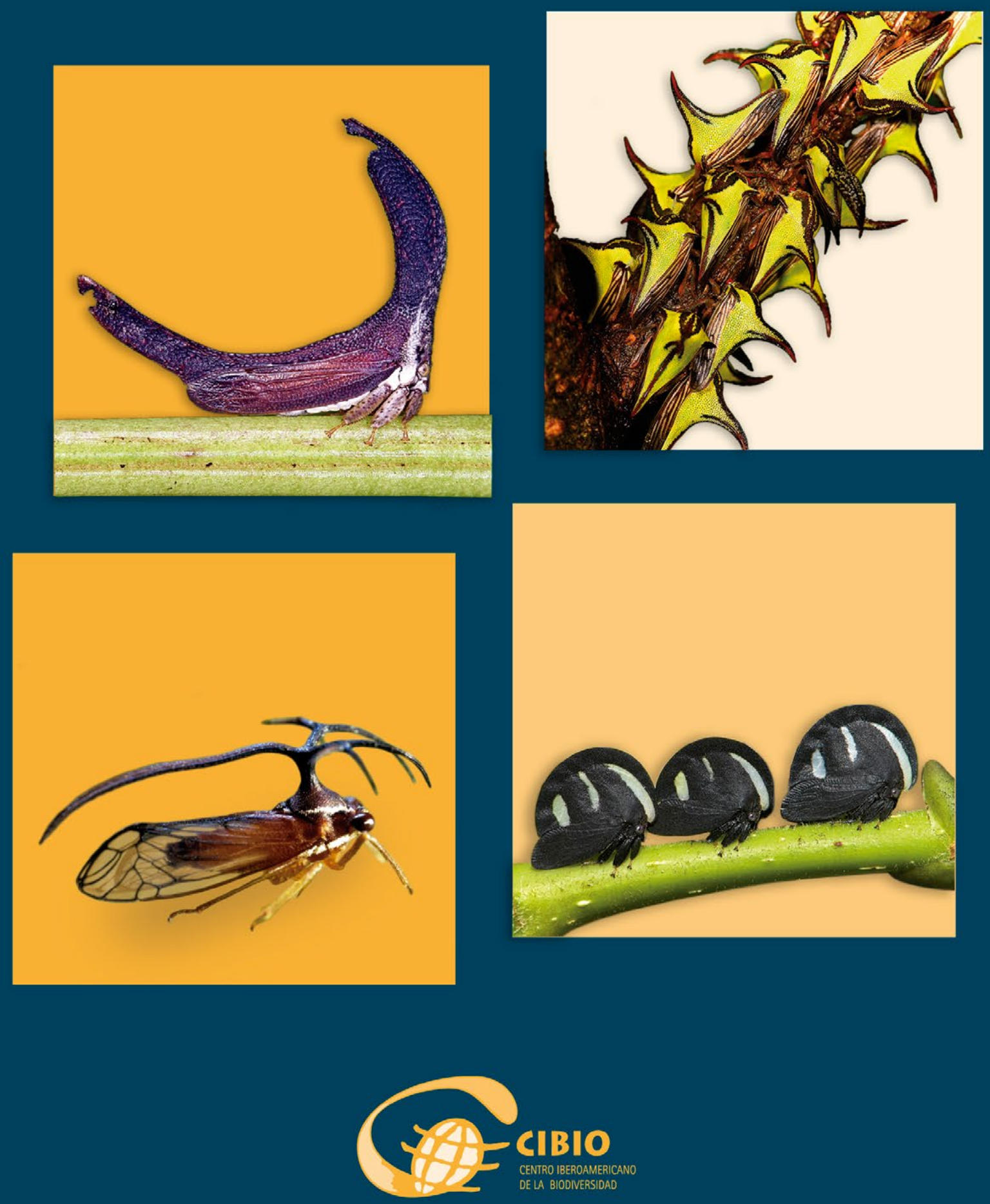

Universitat d'Alacant
Universidad de Alicante 


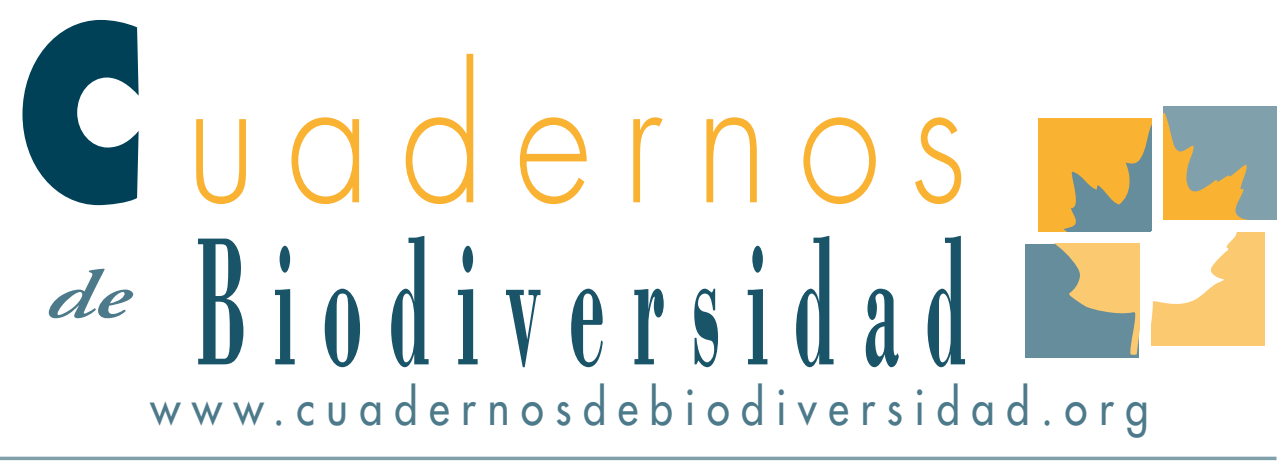

\section{Charcas de vida para el tritón pigmeo y el galápago leproso}

\section{Ulrike Möhring}

Fundación Monte MediterRáneo, ULRIKE.MOEHring@GMX.NET

\section{ABSTRACT}

Ponds are important resources of freshwater; they provide ecologic stepping-stones and have a significant importance as habitats for a multiplicity of plants and animals. But today the European ponds are declining dramatically going along with the disappearing of many species that depend on them.

Fundación Monte Mediterráneo, a foundation for nature conservation and ecologic agriculture, launches a project to rescue the ponds. On the Dehesa San Francisco, an area in Huelva/Andalusia (Spain), still existing ponds will be conserved and optimized and new ponds will be created for the rescue of the aquatic species like the southern marbled newt Triturus pygmaeus (Wolterstorff 1905) an the spanish pond turtle Mauremys leprosa (Schweigger 1812).

Key words: Ponds, amphibians, conservation project, Andalusia, southern marbled newt, Spanish pond turtle

\section{INTRODUCCIÓN}

La pérdida de biodiversidad en flora y fauna, ha alcanzado unos niveles preocupantes. A diario, desaparecen especies en todo el planeta por causas principalmente de origen antropogénico.

Uno de los fines de la Fundación Monte Mediterráneo es la concienciación sobre este fenómeno y, en lo posible, contrarrestar el proceso de pérdida de especies para asegurar un equilibrio ecológico para futuras generaciones. La Dehesa San Francisco tiene unas 700 hectáreas ubicadas en el término municipal de Santa Olalla del Cala (Huelva). Esta dehesa ha sido declarada Reserva Ecológica en 2014, y es propiedad de la Fundación Monte Mediterráneo.

En este artículo se presenta el proyecto de protección de las charcas y sus organismos en la Dehesa San Francisco llevado a cabo por la Fundación Monte Mediterráneo. 


\section{CHARCAS EN PELIGRO}

Los millones de pequeñas masas de agua de menos de 10 hectáreas que existen representan el 30 por ciento de la superficie mundial de agua estancada y tienen una gran importancia ecológica (EPCN 2008). Estos peculiares almacenamientos de agua, representan corredores ecológicos y son esenciales para muchas especies raras y amenazadas. Las charcas temporales y los estanques de la zona mediterránea son uno de los hábitats de agua dulce más destacables y más amenazados de Europa (EPCN 2008).

Según su carácter de fases de inundación y de sequía, las charcas temporales albergan comunidades de plantas y animales muy adaptadas y diversas. El número de las charcas y los estanques en Europa retrocede en una dimensión dramática - en algunos países ha desaparecido hasta un 90 por cien de ellos (EPCN 2008).

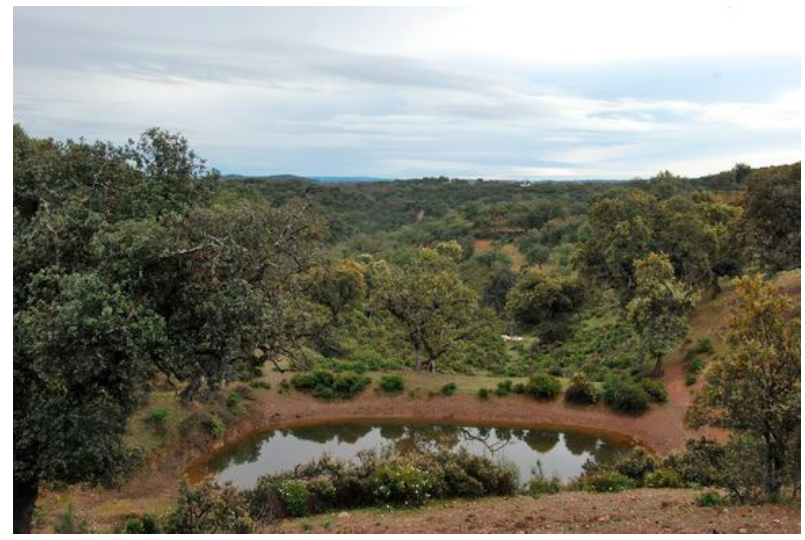

Charca temporal en la parcela Vieja Baja con aspecto primaveral (Foto: U. Möhring).

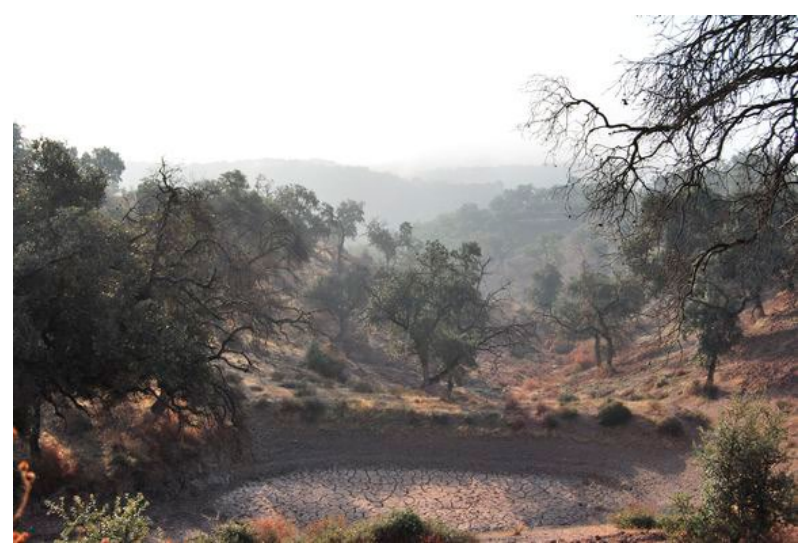

La misma charca en verano tardío. (Foto: U. Möhring).
Sancho y Lacomba (2010) indican algunos de los riesgos y amenazas más importantes para las charcas de la zona mediterránea y sus composiciones peculiares de especies:

- la alteración del hidroperiodo,

- el abandono de prácticas tradicionales,

- la presión ganadera,

- la erosión y la sedimentación,

- la transformación y la intensificación de la agricultura,

- la ocupación por las infraestructuras,

- la pérdida de valor de los puntos de agua,

- el uso recreativo incontrolado,

- la contaminación,

- la introducción de especies exóticas,

- el aislamiento de las poblaciones

- la persecución directa,

- el efecto trampa y

- las enfermedades emergentes, por ejemplo la quitridiomicosis.

En consecuencia y con el avance del cambio climático, concretamente en las regiones con calor estival del sur de España, el número de charcas irá disminuyendo y, con este proceso, se ven especialmente amenazadas aquellas especies cuyo ciclo vital está parcial- o totalmente ligado a la presencia de agua, como por ejemplo los anfibios y los galápagos.

\section{LAS CHARCAS DE LA DEHESA SAN FRANCISCO}

En el terreno de la Dehesa San Francisco en el Parque Natural Sierra de Aracena y Picos de Aroche existe una red de agua dulce en forma de arroyos pequeños y una multitud de charcas temporales, de abrevaderos y de pantanos los cuales albergan un gran número de especies amenazadas y de especial interés comunitario. El origen de la mayoría de las charcas y pantanos es artificial, construido para asegurar el abastecimiento de agua para el ganado. Debido a sus propiedades (p. e. características de su superficie, profundidad, presión ganadera, ciclo 
de alteración de fases de inundación y de sequía, la dimensión del sombreado, tipo de vegetación subacuática y ribereña y la tipo del hábitat terrestre de los alrededores) cada charca mantiene su espectro de especies muy propio debido a la presencia de sus productores y consumidores particulares.

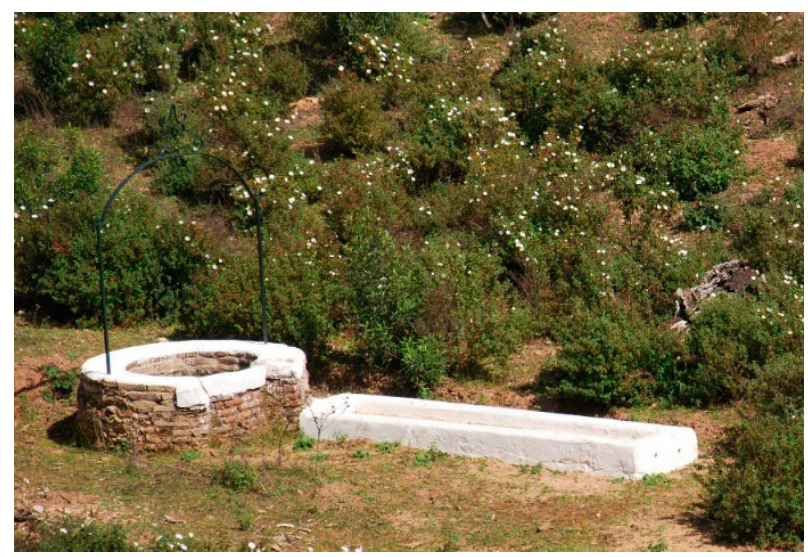

Pozo antiguo con bebedor en la parcela Vieja Baja (Foto: U. Möhring, 2014).

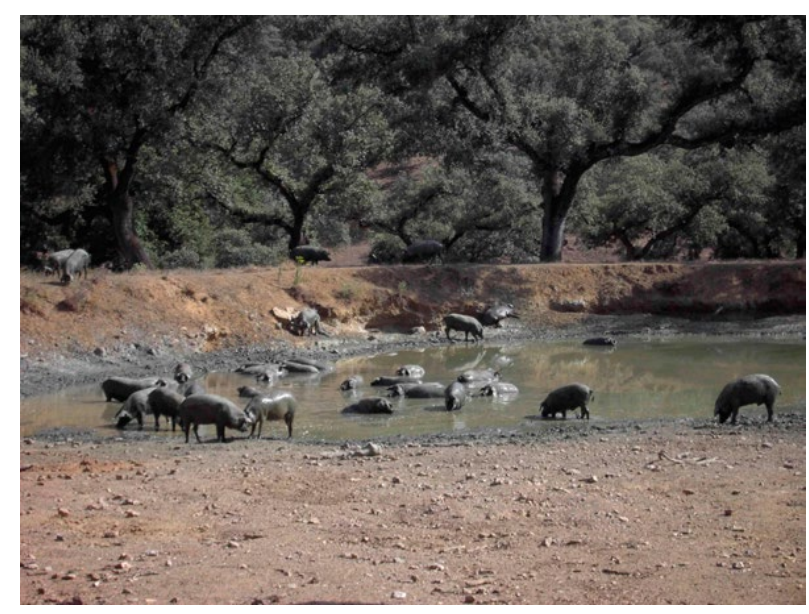

Muchos pantanos de la Dehesa San Francisco tienen una gran importancia para el bienestar de los cerdos ibéricos especialmente durante los veranos calurosos. Pero, debido a la polución por las heces y el pisoteo las charcas de utilidad para el ganado, pierde su gran valor para los animales y plantas acuáticos silvestres (Foto: U. Möhring).

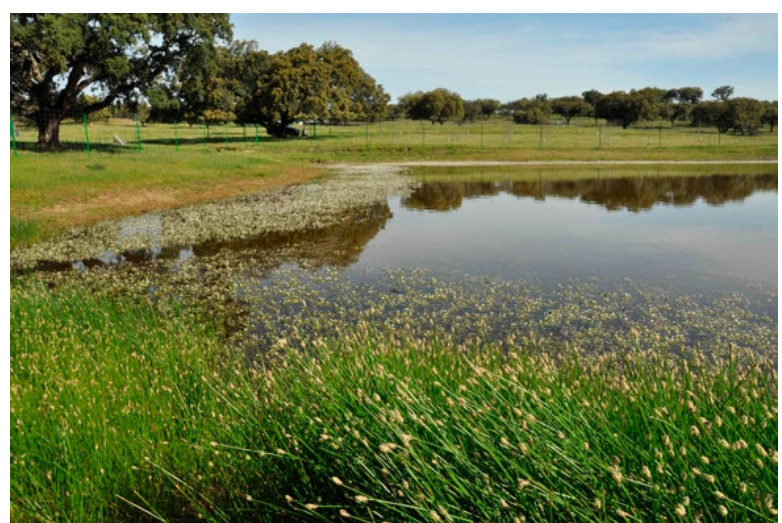

El pantano de Vallebarco está vallado para evitar la intrusión del ganado y contiene una gran abundancia de plantas subacuáticas y ribereñas y forma un hábitat óptimo para los renacuajos del sapo de espuelas Pelobates cultripes (Cuvier 1829), la culebra de agua Natrix maura (Linnaeus 1758) y varios insectos acuáticos (Foto: U. Möhring).

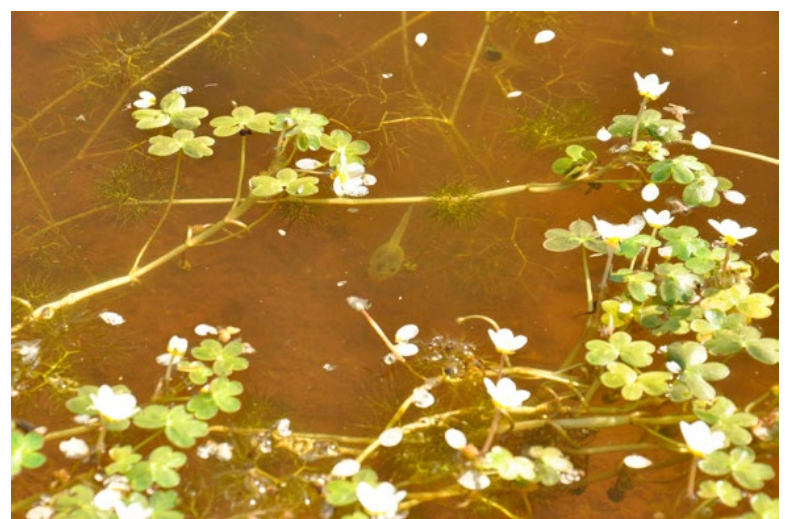

Un renacuajo del sapo de espuelas Pelobates cultripes (Cuvier 1829) entre la vegetación subacuática (ranúnculo Ranunculus sp.) en el pantano de Vallebarco (Foto: U. Möhring).

\section{EL TRITÓN PIGMEO Y EL GALÁPAGO LEPROSO - DOS ESPECIES DE ESPECIAL INTERÉS COMUNITARIO}

Dos especies de especial interés comunitario son el tritón pigmeo (Triturus pygmaeus (Wolterstorff 1905)) el galápago leproso (Mauremys leprosa (Schweigger 1812)).

El tritón pigmeo (Triturus pygmaeus (Wolterstorff 1905)) (libro rojo: Vulnerable VU A2c), durante mucho tiempo, se catalogaba como subespecie del tritón jaspeado y ha sido reconocido como especie 
propia hace poco. La especie está extendida en el suroeste de la península ibérica y habita en bosques y matorrales mediterráneos, arroyos y ríos, charcas y depósitos de agua. Estos animales con actividad nocturna o crepuscular viven durante su fase terrestre principalmente escondidos debajo de ramas o piedras. En otoño, se desplazan a los lugares de desove que deben disponer de agua rica en oxígeno y de abundantes plantas acuáticas. Durante la época de apareamiento, los machos llevan una cresta grande en la espalda. Unos 300 huevos son pegados a las plantas acuáticas. Las larvas, una vez iniciada la transformación, necesitan 3-4 meses para crecer. A principios del verano, abandonan las aguas de desove para pasar los meses calurosos escondidos en el campo. En la Dehesa San Francisco se ha comprobado su presencia en dos pantanos.

Los mayores peligros para el tritón pigmeo son: - pérdida de aguas de desove por desertificación

- fragmentación del paisaje por urbanización y densificación de infraestructura como autovías/autopistas y carreteras

- contaminación de aguas por pesticidas o carga ganadera excesiva

- propagación de especies invasoras como, por ejemplo, el galápago de Florida que son introducidas por ignorancia, desconocimiento y falta de concienciación ecológica.

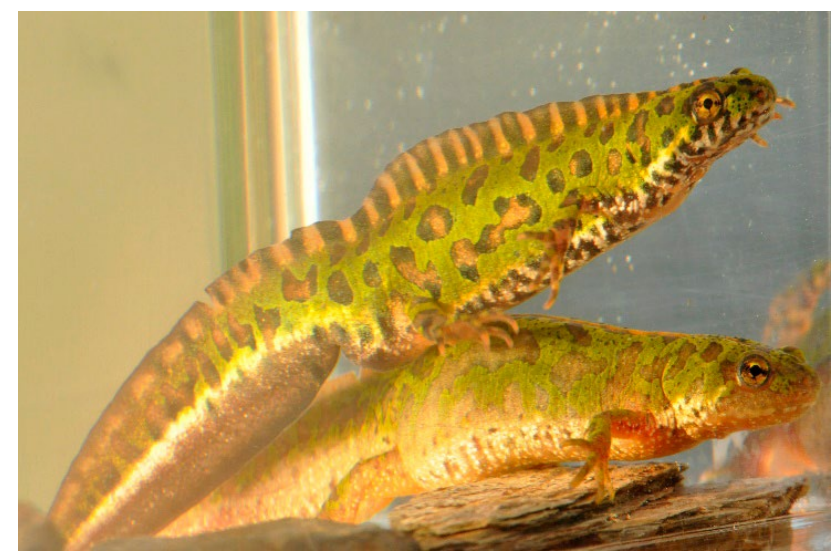

Macho (arriba) y hembra (abajo) de tritón pigmeo (Triturus pygmaeus (Wolterstorff 1905)) (Foto: U. Möhring)

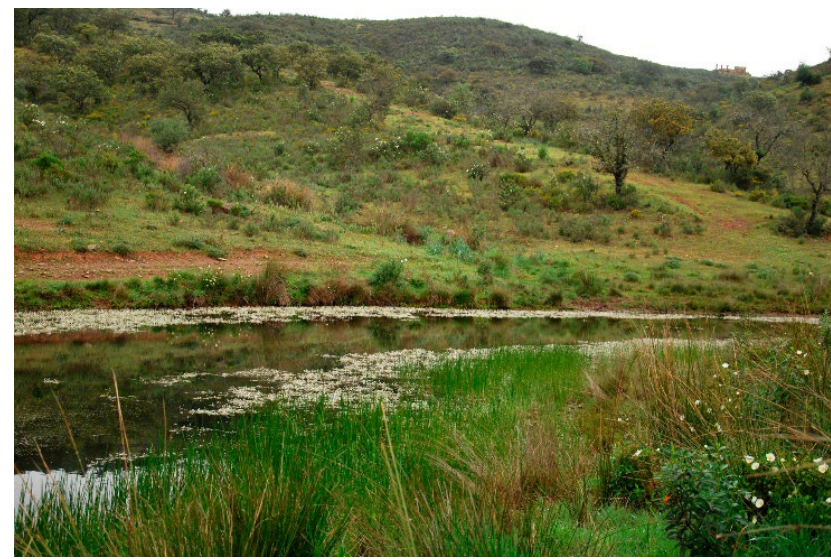

Hábitat ideal para anfibios: Pantano grande en la zona a pie de la Solana del Cuervo: Aguas ricas en oxígeno y con abundancia en plantas acuáticas sumergidas (Foto: U. Möhring).

El galápago leproso (Mauremys leprosa (Schweigger 1812)) (libro rojo: Vulnerable VU A2ac + A3c) es omnívoro y vive siempre en charcas y arroyos. No es muy exigente respecto a la calidad del agua y está extendido en la península ibérica y el norte de África. Aunque se le ve con una cierta frecuencia en la zona, su presencia se ve drásticamente disminuida en determinados puntos debido a:

- un empleo masivo de productos químicos en la agricultura

- la fragmentación de su hábitat

- la desaparición de puntos de agua

- la introducción de especies ajenas como el galápago de Florida

- la caza para consumo.

En la Dehesa San Francisco se le encuentra en dos pantanos y se puede demostrar su reproducción.

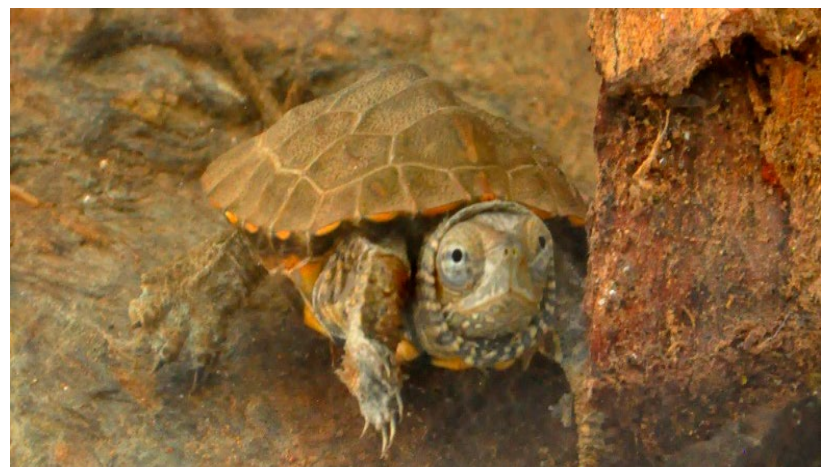

Galápago leproso (Mauremys leprosa (Schweigger 1812)) juvenil (Foto: U. Möhring). 


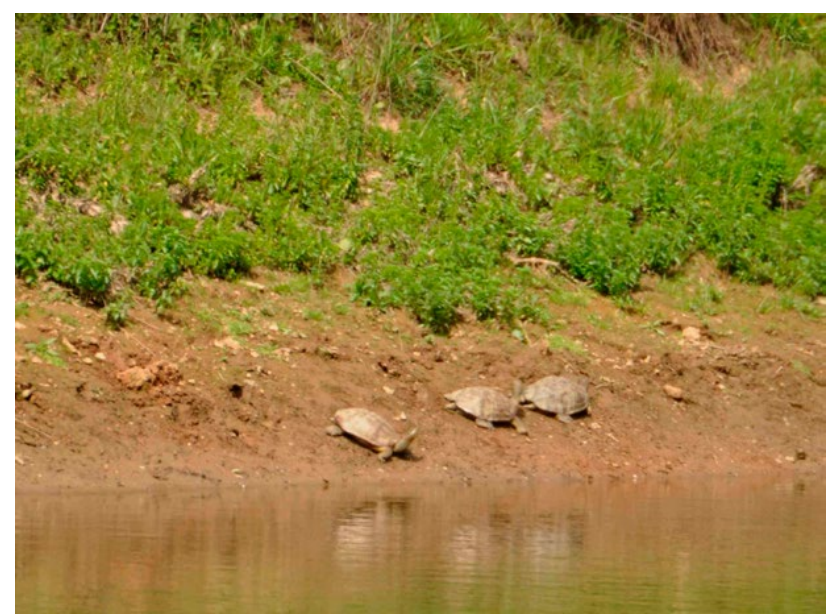

Tres individuos del galápago leproso (Mauremys leprosa (Schweigger 1812)) tomando sol al lado del pantano de Val de Buji (Foto: U. Möhring).

\section{CONTENIDO Y OBJETIVOS DEL PROYECTO}

Con el avance del calentamiento climático global hay que contar con periodos de calor y sequía más intensos y más largos en la zona del mediterráneo que aumentarán la desaparición de hábitats junto a puntos de agua. Ante esta situación alarmante, en la Dehesa San Francisco, se hace necesario tomar las siguientes medidas para la conservación de la población local de especies de anfibios:

- vallar y optimizar charcas temporales e instalar abrevaderos adjuntos
- instalar y vallar nuevas charcas permanentes y temporales adaptadas especialmente a las necesidades de determinadas especies (son especialmente prometedoras aquellas instalaciones de hábitats cuya característica es variada: aguas llanas/profundas, sin/con vegetación en las riberas, cambio del nivel de agua, etc.). Ello garantiza un amplio espectro de hábitats y la probabilidad de ser poblado por muchas de las especies presentes en la finca, sobre todo, porque la finca no está fragmentada por carreteras o urbanizaciones, un seguimiento está previsto.

- plantaciones de setos alrededor de las charcas para optimar los hábitats terrestres y disminuir los procesos de erosión y sedimentación

- medidas de divulgación y concienciación, educación ambiental, material de información y divulgación

- en su caso, construcción de pequeñas pozas de desove

Las mencionadas medidas beneficiarán un gran número de anfibios (véase tabla adjunta) e invertebrados acuáticos, como las libélulas, otros insectos y moluscos cuyo hábitat está relacionado con el agua. Muchos de los anfibios que habitan en la Dehesa San Francisco pertenecen a especies de interés comunitario según la directiva sobre los hábitats naturales y la flora y fauna silvestres - Directiva de Hábitats anexos II y IV. Muchos de ellos se encuentran en las listas rojas y están, por lo menos localmente, en peligro (véase en la tabla 1 ).

Tabla 1: Especies de anfibios presentes en la Dehesa San Francisco (potencialmente o comprobado) y las exigencias de su hábitat acuático.

\begin{tabular}{l|l|l|l|}
\hline \multicolumn{1}{|c|}{$\begin{array}{l}\text { Directiva 92/43/ } \\
\text { CEE de Hábitats: } \\
\text { Anejos }\end{array}$} & Estatus en el Libro rojo & \multicolumn{1}{c|}{ Hábitat acuático } \\
\hline $\begin{array}{l}\text { Salamandra común } \\
\text { Salamandra } \\
\text { salamandra (Linnaeus }\end{array}$ & - & $\begin{array}{l}\text { Nacional: } \\
\text { Casi amenazada (NT) } \\
\text { 1758) } \\
\text { comprobado }\end{array}$ & $\begin{array}{l}\text { Deposición de las larvas en zonas sin flujo } \\
\text { de arroyos pequeños y exento de peces con } \\
\text { agua muy claro, en pozos y en zonas llanas } \\
\text { de lagos o estanques. }\end{array}$ \\
\hline
\end{tabular}




\begin{tabular}{|c|c|c|c|}
\hline Nombre Especie & $\begin{array}{l}\text { Directiva 92/43/ } \\
\text { CEE de Hábitats: } \\
\text { Anejos }\end{array}$ & Estatus en el Libro rojo & Hábitat acuático \\
\hline $\begin{array}{l}\text { Sapo de espuelas } \\
\text { Pelobates cultripes } \\
\text { (Cuvier 1829) } \\
\text { comprobado }\end{array}$ & II, IV & $\begin{array}{l}\text { Nacional: } \\
\text { Preocupación menor (LC) } \\
\text { Especie de interés especial }\end{array}$ & $\begin{array}{l}\text { Desove en charcas temporales, estanques } \\
\text { ricos en vegetación subacuática, a veces en } \\
\text { arroyos de flujo muy lento. }\end{array}$ \\
\hline $\begin{array}{l}\text { Sapillo pintojo ibérico } \\
\text { Discoglossus galganoi } \\
\text { Capula, Nascetti, } \\
\text { Lanza, Bullini \& } \\
\text { Crespo } 1985 \\
\text { potencialmente }\end{array}$ & II, IV & $\begin{array}{l}\text { Nacional: } \\
\text { Preocupación Menor (LC) } \\
\text { Especie de interés especial }\end{array}$ & $\begin{array}{l}\text { En aguas sin y con flujo, en charcas } \\
\text { temporales y permanentes, especialmente en } \\
\text { aguas llanas con mucha vegetación ribereña, } \\
\text { en selvas de vega. }\end{array}$ \\
\hline $\begin{array}{l}\text { Sapillo moteado } \\
\text { ibérico Pelodytes } \\
\text { ibericus Sánchez- } \\
\text { Herraiz, Barbadillo, } \\
\text { Machordom y } \\
\text { Sanchiz } 2000 \\
\text { potencialmente }\end{array}$ & II & $\begin{array}{l}\text { Nacional: } \\
\text { Preocupación Menor (LC) } \\
\text { Especie de interés especial }\end{array}$ & $\begin{array}{l}\text { Desove en charcas llanas y asoleadas con } \\
\text { mucha vegetación subacuática, pozas, fosos. }\end{array}$ \\
\hline $\begin{array}{l}\text { Sapo común Bufo } \\
\text { bufo (Linnaeus 1758) } \\
\text { comprobado }\end{array}$ & - & $\begin{array}{l}\text { Nacional: } \\
\text { Preocupación Menor (LC) }\end{array}$ & $\begin{array}{l}\text { Desove en charcas grandes, estanques y lagos } \\
\text { con una profundidad de } 50 \text { centímetros } \\
\text { como lo mínimo y con áreas grandes exento } \\
\text { de vegetación. }\end{array}$ \\
\hline $\begin{array}{l}\text { Sapo corredor Bufo } \\
\text { calamita Laurenti } \\
1768 \\
\text { comprobado }\end{array}$ & II, IV & $\begin{array}{l}\text { Nacional: } \\
\text { Preocupación Menor (LC) } \\
\text { Especie de interés especial }\end{array}$ & $\begin{array}{l}\text { Desove en charcas y pozas pequeñas y llanas } \\
\text { sin vegetación. }\end{array}$ \\
\hline $\begin{array}{l}\text { Ranita de San Anton } \\
\text { meridional Hyla } \\
\text { meridionalis Boettger } \\
1874 \\
\text { comprobado }\end{array}$ & II, IV & $\begin{array}{l}\text { Nacional: } \\
\text { casi amenaza-da (NT) } \\
\text { Especie de interés especial }\end{array}$ & $\begin{array}{l}\text { Desove en charcas pequeńas ricas en } \\
\text { vegetación subacuática, en pozos, } \\
\text { abrevaderos y albercas. }\end{array}$ \\
\hline $\begin{array}{l}\text { Rana común Rana } \\
\text { perezi Seoane } 1885 \\
\text { comprobado } \\
\end{array}$ & $\mathrm{V}$ & $\begin{array}{l}\text { Nacional: } \\
\text { Preocupación Menor (LC) }\end{array}$ & $\begin{array}{l}\text { En varios tipos de agua como charcas, } \\
\text { estanques, lagos, albercas, abrevaderos, } \\
\text { humedales y ríos. }\end{array}$ \\
\hline \begin{tabular}{|l|} 
Sapo partero ibérico \\
Alytes cisternasii Boscá \\
1879 \\
potencialmente
\end{tabular} & II, IV & $\begin{array}{l}\text { Nacional: } \\
\text { casi amenaza-da (NT) } \\
\text { Especie de interés especial }\end{array}$ & $\begin{array}{l}\text { Deposición de los renacuajos en charcas y } \\
\text { arroyos pequeños. }\end{array}$ \\
\hline $\begin{array}{l}\text { Gallipato Pleurodeles } \\
\text { waltl Michahelles } \\
1830 \\
\text { comprobado }\end{array}$ & II & $\begin{array}{l}\text { Nacional: } \\
\text { casi amenaza-da (NT) } \\
\text { Especie de interés especial }\end{array}$ & $\begin{array}{l}\text { En varios tipos de agua sin flujo y con flujo } \\
\text { lento. }\end{array}$ \\
\hline
\end{tabular}




\begin{tabular}{|c|c|c|c|}
\hline Nombre Especie & $\begin{array}{l}\text { Directiva 92/43/ } \\
\text { CEE de Hábitats: } \\
\text { Anejos }\end{array}$ & Estatus en el Libro rojo & Hábitat acuático \\
\hline $\begin{array}{l}\text { Tritón ibérico Triturus } \\
\text { boscai (Lataste 1879) } \\
\text { potencialmente }\end{array}$ & II & $\begin{array}{l}\text { Andalucía: Riesgo Menor: } \\
\text { casi amenaza-da (NT) } \\
\text { Nacional: } \\
\text { Preocupación Menor (LC) } \\
\text { Especie de interés especial }\end{array}$ & $\begin{array}{l}\text { En charcas pequeñas con agua muy claro, } \\
\text { pozos y arroyos pequeños con agua claro y } \\
\text { fresco. }\end{array}$ \\
\hline $\begin{array}{l}\text { Triton pigmeo } \\
\text { Triturus pygmaeus } \\
\text { (Wolterstorff 1905) } \\
\text { comprobado }\end{array}$ & II, IV & $\begin{array}{l}\text { Nacional: } \\
\text { Vulnerable (VU) } \\
\text { Especie de interés especial }\end{array}$ & $\begin{array}{l}\text { En varios tipos de agua sin flujo y con } \\
\text { mucha vegetación subacuática como charcas, } \\
\text { estanques y abrevaderos. }\end{array}$ \\
\hline
\end{tabular}

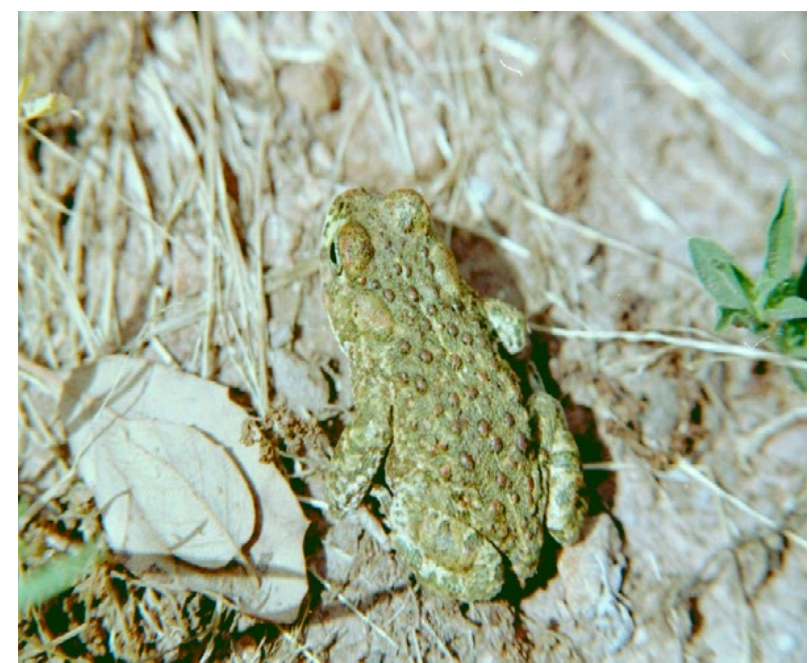

El sapo corredor Bufo calamita Laurenti 1768 es una especie "pionera" que desova en pozas temporales como huellas de ruedas en los caminos

(Foto: U. Möhring, Dehesa San Francisco 2002).

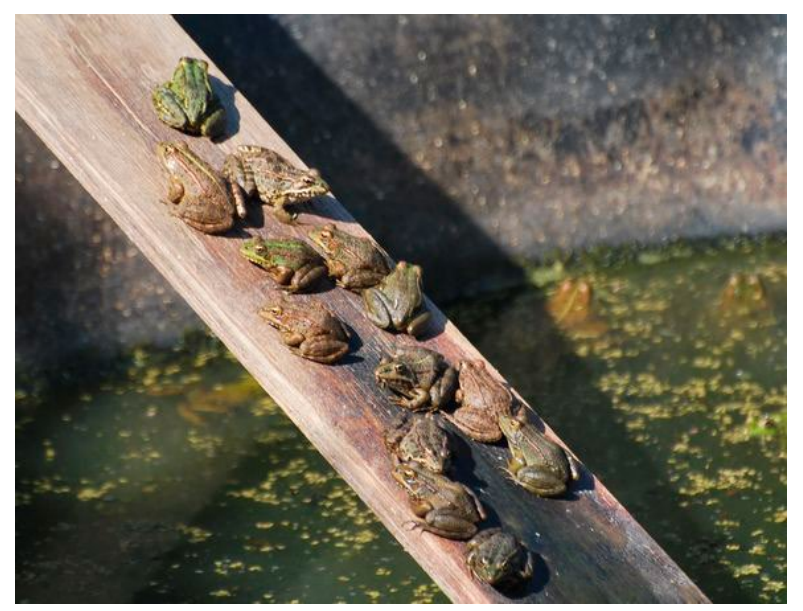

Un grupo de la Rana común Rana perezi Seoane 1885 en una charca de la parcela de San Francisco. La especie está ligada al agua durante el año entero (Foto: U. Möhring, 2011).

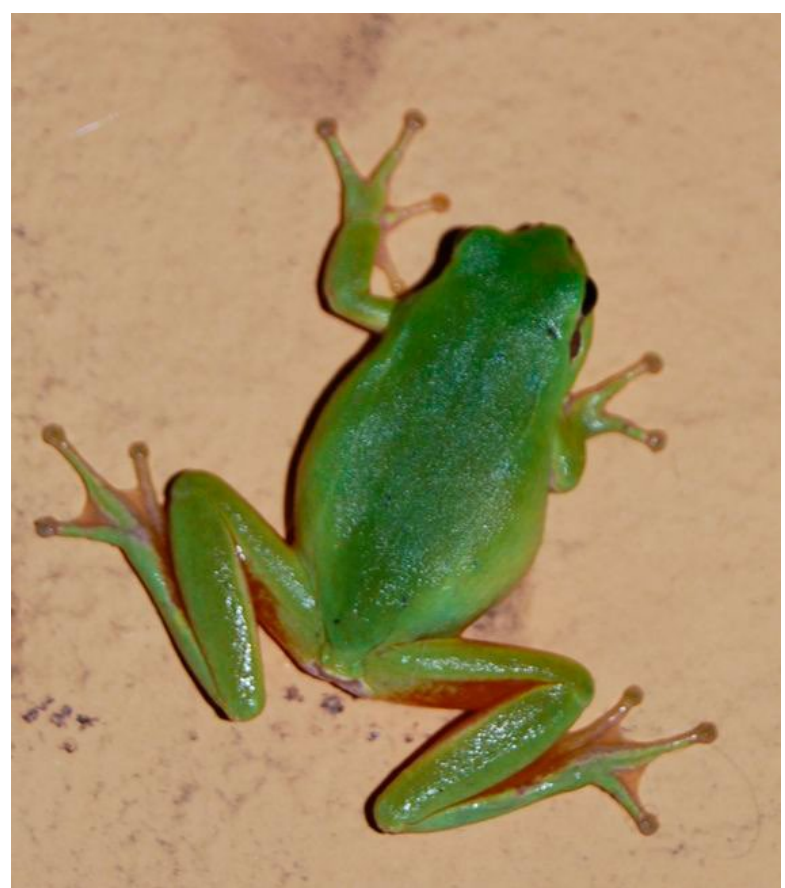

La Ranita de San Antón meridional Hyla meridionalis Boettger 1874 necesita un hábitat de abundantes plantas acuáticas para desove (Foto: U. Möhring).

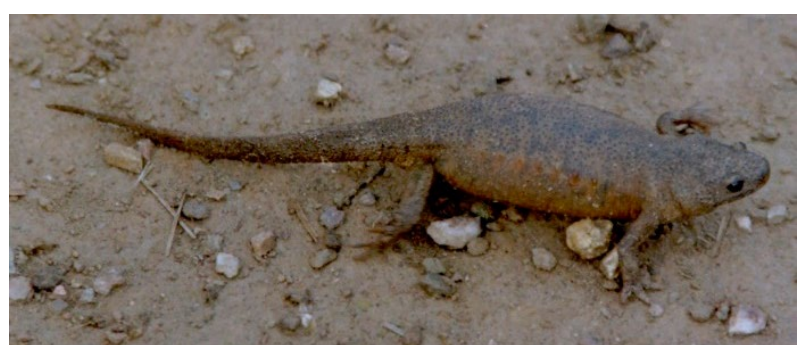

El gallipato Pleurodeles waltl Michahelles 1830, aquí en la forma terrestre, no es muy exigente respecto a la calidad del agua. La especie vive acuático y terrestre, pero algunos individuos no salen del agua durante el año entero (Foto: U. Möhring, San Francisco 2001). 


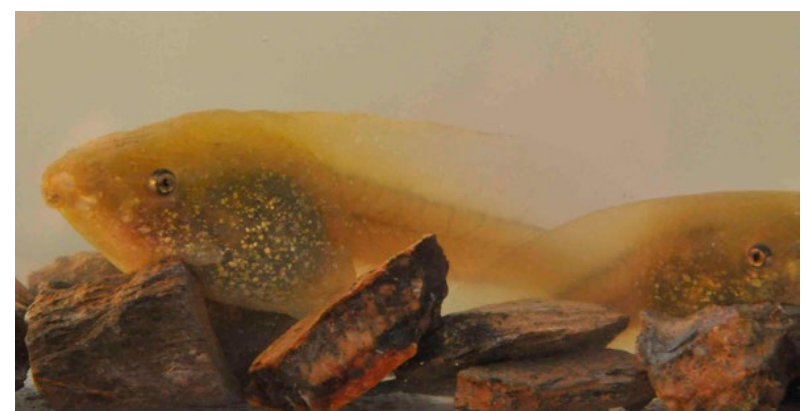

Los renacuajos del sapo de espuelas Pelobates cultripes (Cuvier 1829) logran un tamaño extraordinario y sobresalen los adultos. El desove tiene lugar en charcas ricas de plantas subacuáticas (Foto: U. Möhring).

Aparte de ello, los anfibios suponen una fuente de alimentación para varias especies vertebradas: las cigüeñas blancas y negras Ciconia ciconia (Linnaeus 1758) y Ciconia nigra (Linnaeus 1758), el milano negro Milvus migrans (Boddaert 1783), la culebra de agua Natrix maura (Linnaeus 1758), la culebra de collar Natrix natrix (Linnaeus, 1758), y, con ellos, también el águila culebrera Circaetus gallicus (Gmelin 1788) se beneficiarían de las medidas. Además, las charcas forman hábitats alimenticios para una multitud de aves, por ejemplo el andarríos chico Actitis hypoleucos (Linnaeus 1758) o el chorlitejo común Charadrius dubius Scopoli 1786, los cuales buscan animales del bentos en las orillas, y los ánades reales Anas platyrhynchos Linnaeus 1758 que son omnívoros, se alimentan de las plantas acuáticas y de la fauna acuática invertebrada muy rica.

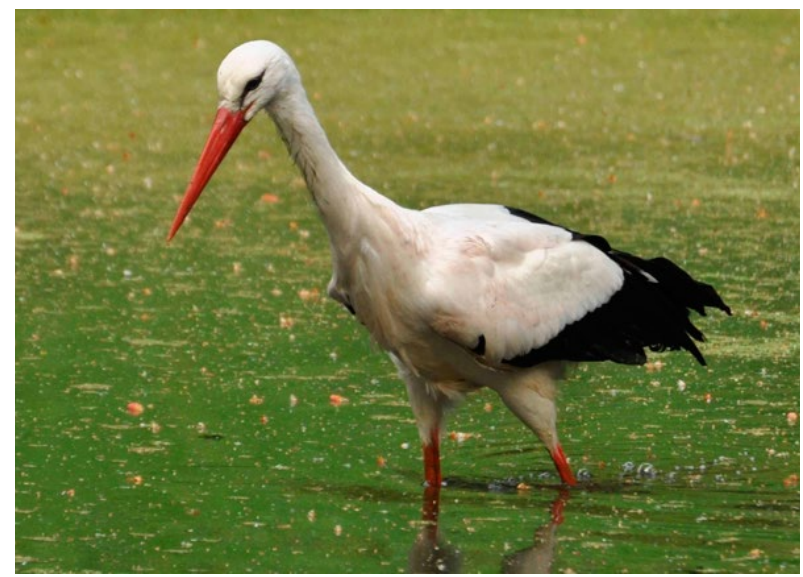

Algunas parejas del cigüeña blanca Ciconia ciconia (Linnaeus 1758) anidan en Santa Olalla del Cala. Para la ingesta visitan con frecuencia las charcas de la Dehesa San Francisco (Foto: U. Möhring).

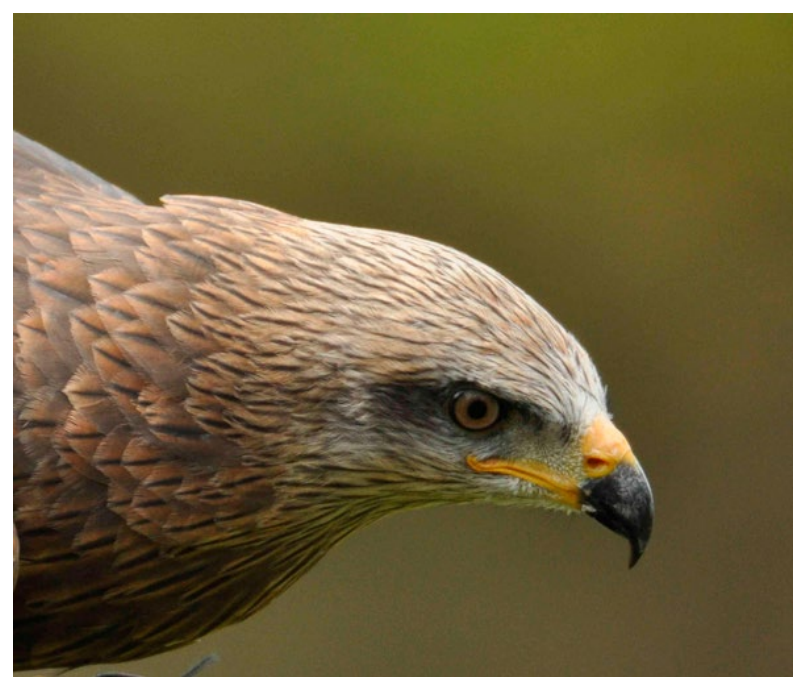

El milano negro Milvus migrans (Boddaert 1783) suele criar cerca del agua (Gacio Iovino \& Hernández Mancha 2006) y caza periódicamente al lado del pantano de la parcela Val de Buji (Foto: U. Möhring).

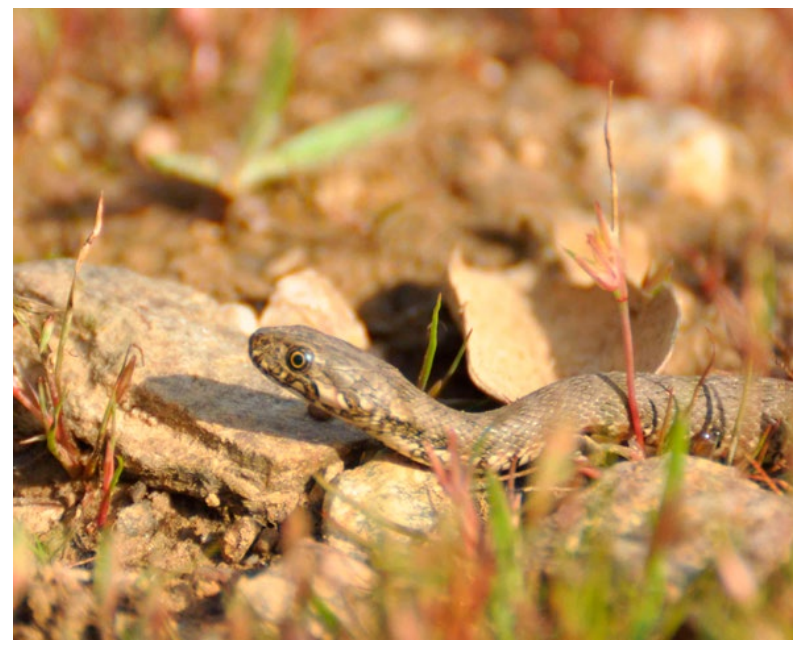

La culebra de agua Natrix maura (Linnaeus 1758) es una serpiente muy frecuente en la Dehesa san Francisco. La culebra es una buceadora y nadadora perfecta que prefiere cazar peces pequeños (Kwet 2010). En la Dehesa San Francisco se alimenta sobre todo de los anfibios y sus larvas (Foto: U. Möhring). 


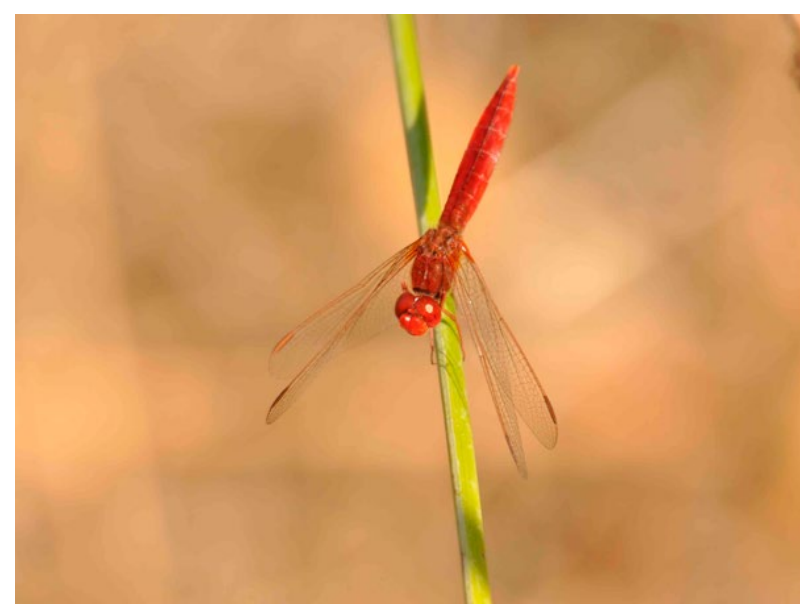

Crocothemis erythraea (Brullé 1832) se reproduce en varias charcas soleadas (Dijkstra 2014). En la Dehesa San Francisco se reproduce por ejemplo en el Pantano de la Solana del Cuervo (Foto: U. Möhring).

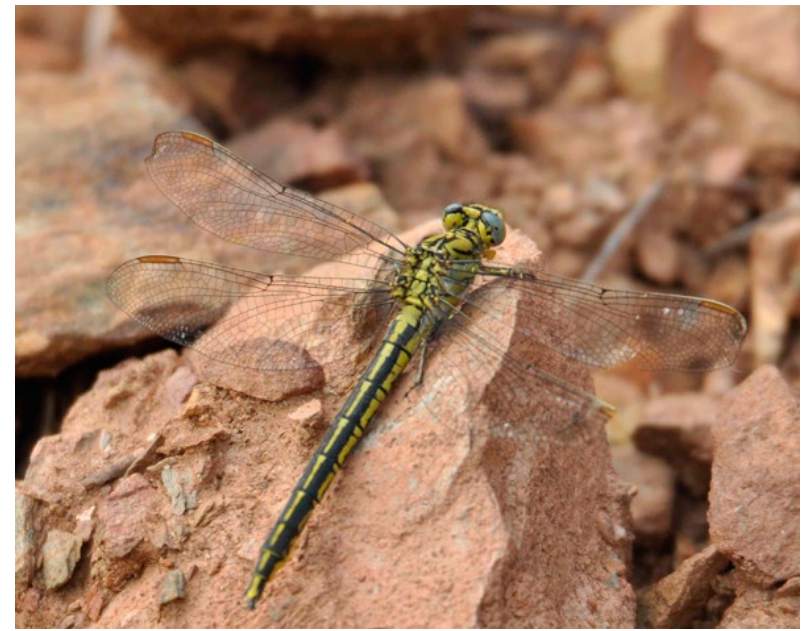

Gomphus pulchellus Selys 1840 tolera una corriente módico (Dijkstra 2010). Se reproduce también en el Pantano de la Solana del Cuervo (Foto: U. Möhring).

\section{REFERENCIAS}

Dijkstra KDB. 2014. Libellen Europas - Der Bestimmungsführer. Haupt. Bern. 320 pp.

EPCN - European Pond Conservation Network. 2008. Manifiesto sobre las charcas. $19 \mathrm{pp}$.

Fernández-Dardenete JR et al. 2013. Guia de los anfibios del sureste ibérico. Parrondo-Celdrán, P. (coord.). Murcia. Asociación Columbares. 176 pp.

Gacio Iovino H \& Hernández Mancha R. 2006. Guía de las Aves del Parque Natural Sierra de Aracena y Picos de Arroche. Sevilla. 248 pp.

Kwet A. 2010. Amphibien und Reptilien Europas. FranckhKosmos-Verlags-Gmbh \& Co. KG. Stuttgart. 252 pp.

MAGRAMA - Ministerio de Agricultura, Alimentación y Medio Ambiente: Listado de Especies de Anfibios de Interés Especial, de http://www.magrama.gob.es/. Fecha de la descarga 13/05/2014

Pleguezuelos J M, Márquez R \& Lizana M. 2002. Atlas y Libro Rojo de los Anfibios y Reptiles de España. Dirección General de Conservación de la NaturalezaAsociación Herpetologica Espańola (2a impresión), Madrid, 587 pp.

Pro natura 2014. Temporäre Gewässer für Amphibien schaffen - Leitfaden für die Praxis, Beiträge zum Naturschutz in der Schweiz, Nr. 35/2014, Basel, 46 pp.

Sancho V \& Lacomba I. 2010. Conservación y Restauración de puntos de agua para la biodiversidad. Colección Manuales Técnicos de Biodiversidad, 2. Generalitat. Conselleria de Medi Ambient, Aigua, Urbanisme i Habitatge. 168 pp.

Zumbach S \& Ryser J. 2012. Weiherbau, Bern, 19 pp. 